\title{
Performance of CDMA System Using m-ZCZ Sequences
}

\author{
M.Jayasheela \\ SNS College of Technology \\ Vazhiyampalayam \\ Coimbatore-641035 \\ $+91-9789300500$ \\ chrislatalus@yahoo.co.in
}

\author{
A.Rajeswari \\ Coimbatore Institute of Technology \\ Peelamedu \\ Coimbatore-641014 \\ $+91-9865907804$ \\ rajeswari.ece.cit@gmail.com
}

\begin{abstract}
Spreading Sequences are used in Code Division Multiple Access for spreading the data for antijamming, anitinteference, low probability of intercept, multiple user access communication and high resolution ranging. Most commonly used spreading Sequences are Maximal Length and Zero Correlation Zone Sequences code called m-ZCZ Sequences. ZCZ Sequences have both autocorrelation side lobes, cross correlation function are zero and sequence length is flexible. In this paper, a comparison of Bit Error Rate performance of Direct Sequence Code Division Multiple Access using Maximal Sequences and Zero Correlation Zone Sequences in AWGN and Rayleigh fading channel is presented. From the results it is seen that m-ZCZ Sequences exhibit better Bit Error Rate Performance compared to that of Maximal Length sequences.
\end{abstract}

\section{Categories and Subject Descriptors}

I.6Simulation and Modeling:I.6.6: Simulation Output Analysis and G.4 MATLAB

\section{General Terms}

Performance.

\section{Keywords}

CDMA, Spread Spectrum Communications, Maximal Length Sequences, Zero Cross Correlation Zone Sequences, Correlation, Bit Error Rate.

\footnotetext{
Permission to make digital or hard copies of all or part of this work for personal or classroom use is granted without fee provided that copies are not made or distributed for profit or commercial advantage and that copies bear this notice and the full citation on the first page. To copy otherwise, or republish, to post on servers or to redistribute to lists, requires prior specific permission and/or a fee. Mobimedia'09, September 7-9, 2009, London, UK. Copyright 2009 ICST 978-963-9799-62$2 / 00 / 0004 \ldots \$ 5.00 "$
}

\section{INTRODUCTION}

The major concern in Wireless communication is efficient use of Bandwidth and power. Spread-spectrum techniques are methods by which energy generated in a particular bandwidth is deliberately spread in the frequency domain, resulting in a signal with a wider bandwidth. This technique decreases the potential interference to other receivers while achieving privacy. Spread spectrum generally makes use of a sequential noise-like signal structure to spread the normally narrow band information signal over a relatively wide band of frequencies. These signals are then collected onto their original frequency at the receiver, and are very inconspicuous as to be transparent. The receiver correlates the received signals to retrieve the original information signal.

The commonly used spread sequences on Direct Sequence CDMA System are Maximal Length Sequences (msequences)[4]. The important disadvantages of m-Sequences are poor cross correlation. Recent literature presents a class of mZCZ sequences which show better cross correlation property compared to that of m-sequences[2].In this paper, a performance comparison of CDMA System using m-Sequences and m- ZCZ Sequences is presented.

The rest of paper is organized as follows; Section 2 deals with System Model. Section 3 presents the Simulation Model of CDMA System. Simulation Results and Discussion are given in Section 4.

\section{SYSTEM MODEL}

A direct sequence spread spectrum system achieves its spreading capability by modulating a narrow band data using a wide band pseudo noise Sequences [4]. The system uses orthogonal codes or uncorrelated codes unique to each user so that all users will be able to transmit their message simultaneously in the same frequency band.The signal of each user will have very low power because of spreading and will appear to be background noise to all other users. The desired user's signal is detected at the receiver using the same spreading code used to encode the base band signal at the transmitter. All other users are interferers to the desired users. The interference due to the undesired users is called Multiple Access Interference (MAI).This interference can be minimized by proper power control at the base station. In the absence of power control, the strongest received signal through not the desired one is picked 
up and detected by the base station thus increasing the probability of error [6].

The capacity of a single cell CDMA system in terms of the number of users is given by

$$
C=1+\frac{\left(\frac{W}{R}\right)}{\left(\frac{E b}{N o}\right)}-\frac{N B}{S}
$$

where S denotes the desired users' power

$\mathrm{N}_{\mathrm{B}}$ denotes the background noise power

$\mathrm{W}$ denotes the total bandwidth

$\mathrm{R}$ denotes the information data rate

$\mathrm{E}_{\mathrm{b}}$ denotes the energy density of the transmitted data

$\mathrm{N}_{\mathrm{o}}$ is the power spectral density of total noise and interference

The parameter $E_{b} / N_{o}$ is defined as the value required for adequate performance to get Bit Error Rate (BER) of $10^{-3}$ or better for systems with digital voice transmission.

The capacity is also given in terms the Signal to Noise Ratio (SNR) by

$$
C=1+\frac{1}{\frac{S}{N}}-\frac{N B}{S}
$$

where $\mathrm{S} / \mathrm{N}$ is the SNR and it is the ratio of the Signal power to the Noise power. The capacity is decreased by the inverse of the SNR.Thus CDMA is a multiple access scheme which has a limit on the number of users that the system can accommodate.

\section{$2.1 \mathrm{~m}$-Sequences}

PN sequences are generated by a Linear Feedback Shift Register (LFSR). A sequence generated by an LFSR with $r$ shift registers is said to be a maximal length sequence or m-Sequences if its length is $\mathrm{N}=2^{\mathrm{r}}$-1.The period of this sequence is the possible number of states that an LFSR can take except an all zero state.The feedback connections are determined by the generator polynomial $\mathrm{g}(\mathrm{D})$ which is a primitive polynomial Peterson R.L et al (1995). A LFSR for generating the PN Sequences is shown in Figure 1.

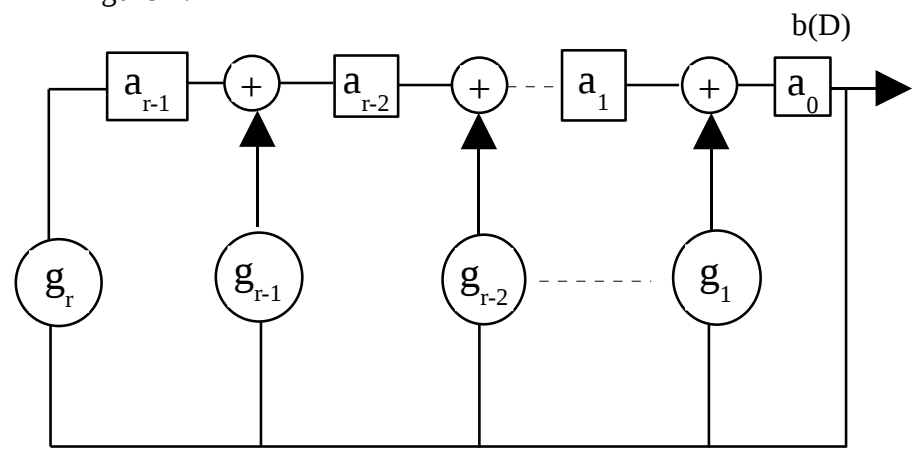

Figure 1: PN Sequence Generator

In Figure $1, \mathrm{a}_{\mathrm{r}-1}, \ldots ., \mathrm{a}_{\mathrm{o}}$ denote the shift registers. The feedback connections are determined by the coefficients $\mathrm{g}_{\mathrm{r}}, \mathrm{g}_{\mathrm{r}-1}, \ldots, \mathrm{g}_{1}$.
The properties of m-sequences are given below:

Property 1: An m-sequence contains, one more one, than zero.

The number of ones is $(\mathrm{N}+1) / 2$.

Property 2: A run is defined as a sequence of a single type of binary digits within the m-sequence.The length of this subsequence is the length of the run. For an m-Sequences, there are 1 run of ones of length $r, 1$ run of zeros of length $r-1,1$ run of ones of length $\mathrm{r}-2$ \& 1 run of zeroes of length $\mathrm{r}-2,2$ runs of ones of length r-3 \& 2 runs of zeros of length r-3,4 runs of ones length $r-4, \ldots \ldots \ldots \ldots \ldots, 2^{r-3}$ runs of ones of length $1 \& 2^{r-3}$ runs of zeros of length 1 .

Property 3: The periodic autocorrelation function $\theta_{b}(k)$ of an mSequences is two valued and is given by

$$
\theta_{b}(k)= \begin{cases}1 & k=I N \\ -1 / N & k \neq I N\end{cases}
$$

Where $l$ is any integer and $\mathrm{N}$ is the period of the Sequences.

Property4: Let $b(D)$ represents a m-Sequence. Let this sequence be shifted by $\mathrm{j}$ chips represented by $\mathrm{b}(\mathrm{D}+\mathrm{j})$. Then the modulo- 2 sum of the two sequences results in a sequence $b(D+k)$ which is the same m-sequence shifted by k chips.

\section{$2.2 \mathrm{~m}-\mathrm{ZCZ}$ Sequences}

$\mathrm{ZCZ}$ codes used in CDMA system is interference free as long as the one-side ZCZ length longer than the maximum single delay spread, which is caused by multipath propagation or/and asynchronous transmission[2].The ZCZ length of conventional binary ZCZ code is restricted to $2^{\mathrm{k}}$, where $\mathrm{k}$ is an integer. Therefore, in practical applications the ZCZ length has to be chosen much larger than required. As a result the number of available codes is limited, which is inversely proportional to the ZCZ length. A binary ZCZ code based on m-sequences, called m-ZCZ code. Its length can be flexibly selected to match the maximum time delay with the great degree-of-freedom, such that constructed interference resistant CDMA system can support more users than that using conventional binary ZCZ codes..

\subsubsection{Generation}

The $\mathrm{m}-\mathrm{ZCZ}$ code set is denoted as $\mathrm{m}$ $\mathrm{ZCZ}\left(\mathrm{C}_{\mathrm{L}}, \mathrm{S}, \mathrm{W}_{\text {min }}\right)=\left\{\left(\mathrm{Z}_{\mathrm{i} 1}, \mathrm{Z}_{\mathrm{i} 2}\right)\right\}$, where $\mathrm{i}=0,1 \ldots \mathrm{S}-1, \mathrm{C}_{\mathrm{L}}$ represents code length, $S$ - set size of the code set, $\mathrm{W}_{\min }-$ minimum length of one-side ZCZ.

The first sub-code of the $\mathrm{i}^{\text {th }}$ code is $\mathrm{Z}_{\mathrm{i} 1}=a^{i . w \text { min }}$ with the length of $\mathrm{N}$, where $\mathrm{a}^{0}$ is an m-sequence with its period $\mathrm{N}$, and $a^{i . w \min }$ stands for the sequence generated by cyclically leftward shifted $\mathrm{a}^{0}$ with i. $\mathrm{W}_{\min }$ chips. This can be represented as

$a_{n}^{i . w \min }=a_{(n+i . w \min )}^{o} \bmod N$

where $n=0,1, \ldots . . N-1$ and $n$ is the chip index.

The second sub-code $\mathrm{z}_{\mathrm{i} 2}=\{+1\}$ contains only one “+1" chip. The congregated code length is $\mathrm{C}_{\mathrm{L}}=\mathrm{N}+1$. The set size of the code is $\mathrm{S}=\left[\mathrm{N} / \mathrm{W}_{\min }\right]$.

The periodic auto-correlation function (ACF) of an m-ZCZ code and periodic cross- correlation function (CCF) of any two mZCZ codes can be calculated as follows: 


$$
\begin{aligned}
& R i, j(k)=\sum_{n-0}^{N-1} a_{n}^{i . w \min } a_{n+k}^{j . w \min }+z_{i 2} z_{j 2} \\
& =\sum_{n=0}^{N-1} a_{(n+i . w \min )}^{0} \bmod N a_{(n+1+j . w \min )}^{0} \bmod N+z_{i 2} Z_{j 2} \\
& =\left\{\begin{array}{ccc}
N+1 & i=j, & k=0 \\
-1+1 & i=j, 0<|k|<w_{\min } & \text { or } \quad i \neq j,|k|<W \text { min }
\end{array}\right. \\
& =\left\{\begin{array}{ccc}
C_{L} & i=j, & k=0 \\
0 & i=j, 0<|k|<w_{\min } \text { or } & i \neq j, \quad|k|<w \min
\end{array}\right.
\end{aligned}
$$

where integer $\mathrm{k}$ denotes the relative time shift.

\subsubsection{Correlation property}

From equation (5) it is seen that the ACF of any m-ZCZ code is zero when $0<|k|<\mathrm{W}_{\min }$ and the CCF between any two codes is zero $|k|<\mathrm{W}_{\text {min. }}$.Thus, there exists a ZCZ with minimum one-side length being $\mathrm{w}_{\min }$. The $\mathrm{w}_{\min }$ can be flexibly controlled by adjusting the number of cyclic shifted chips.

\section{Example:}

Given $\mathrm{L}=64, \mathrm{w}_{\min }=30$ a set of $\mathrm{m}-\mathrm{ZCZ}$ codes are denoted by $(64,2,30)$ containing $\mathrm{J}=[63 / 30]=2$ codes as

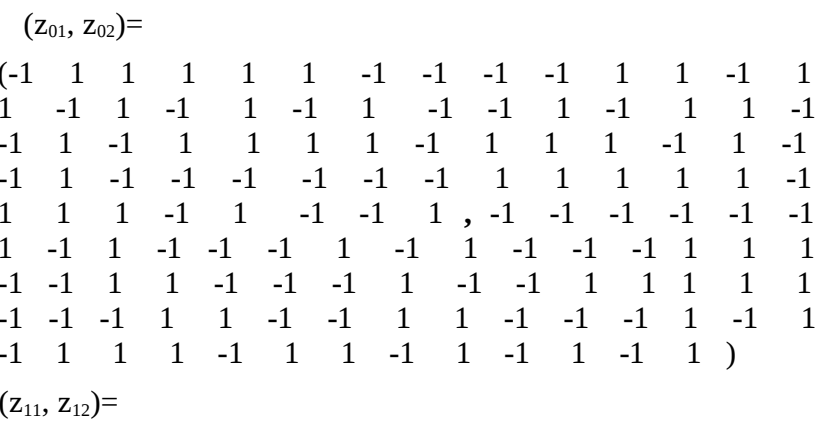

$\left(\begin{array}{llllllllllllll}-1 & 1 & 1 & 1 & 1 & 1 & -1 & -1 & -1 & -1 & 1 & 1 & -1 & 1\end{array}\right.$ $\begin{array}{llllllllllllll}1 & -1 & 1 & -1 & 1 & -1 & 1 & -1 & -1 & 1 & -1 & 1 & 1 & -1\end{array}$ $\begin{array}{lllllllllllllll}-1 & 1 & -1 & 1 & 1 & 1 & 1 & -1 & 1 & 1 & 1 & -1 & 1 & -1 & -1\end{array}$ $\begin{array}{llllllllllllll}1 & -1 & -1 & -1 & -1 & -1 & -1 & 1 & 1 & 1 & 1 & 1 & -1 & 1\end{array}$ $\begin{array}{lllllllllllllll}1 & 1 & -1 & 1 & -1 & -1 & 1 & , & -1 & -1 & -1 & -1 & -1 & -1 & 1\end{array}$ $\begin{array}{lllllllllllllll}-1 & 1 & -1 & -1 & -1 & 1 & -1 & 1 & -1 & -1 & -1 & 1 & 1 & 1 & -1\end{array}$ $\begin{array}{lllllllllllllll}-1 & 1 & 1 & -1 & -1 & -1 & 1 & -1 & -1 & 1 & 1 & 1 & 1 & 1 & -1\end{array}$ $\begin{array}{lllllllllllllll}-1 & -1 & 1 & 1 & -1 & -1 & 1 & 1 & -1 & -1 & -1 & 1 & -1 & 1 & -1\end{array}$ $\begin{array}{lllllllllllll}1 & 1 & 1 & -1 & 1 & 1 & -1 & 1 & -1 & 1 & -1 & 1 & \text { ) }\end{array}$

\subsubsection{Parameters}

When $\mathrm{w}_{\min }=1, \mathrm{~S}=\left[\mathrm{N} / \mathrm{w}_{\min }\right]=\mathrm{N}$, the $\mathrm{m}-\mathrm{ZCZ}$ code set is equal to Walsh Hadamard code set excluding the all-one code for the given code length. When $\mathrm{w}_{\min }=\mathrm{N}$, then $\mathrm{ZCZ}$ length reaches its maximum value and there is only one code in this set, whose ACF has a ZCZ with one-side length $\mathrm{N}$. When $1<\mathrm{w}_{\min }<\mathrm{N}$, the set size $\mathrm{S}$ satisfies $1<\mathrm{S}=\left[\mathrm{N} / \mathrm{w}_{\min }\right]<\mathrm{N}$. Therefore, length of $\mathrm{m}$ $\mathrm{ZCZ}$ can be increased by reducing the set size.

\section{3 m-ZCZ Sequences in CDMA System}

The advantage of CDMA systems is that channel capacity is increased by 3 times compared to FDMA and TDMA techniques..In CDMA technique, all users share the same radio channel simultaneously. When users are idle, the MAI is greatly reduced by $65 \%$. CDMA has a soft capacity limit because addition of new users to a CDMA system will only cause a graceful degradation of signal quality. All users are different only in PN codes. Hence there is no handoff from one frequency to another.

Spreading code is formed at the transmitter side by two subcodes of an m-ZCZ code with a cyclic prefix and a cyclic postfix inserted at both sides of each sub-code. The lengths of the cyclic prefixes and cyclic postfixes should be at least equal to the maximum multipath time delay, so that the two sub codes will not overlap with each other after being transmitted via multipath channels.

Despreading code is formed at the receiver side by the two subcodes of an m-ZCZ code, with zeroes inserted in the guard intervals of identical length which should be equal to that of the cyclic prefixes and postfixes in the spreading code. To make decisions, aperiodic correlation is performed between the received signal and the local despreading code. Due to the effects of cyclic prefix and postfix the aperiodic correlation automatically becomes periodic correlation.The proposed $\mathrm{m}$ ZCZ code could be more suitable for wireless systems with a relatively short delay spread.

Spreading Code

\begin{tabular}{|l|l|l|l|l|l|}
\hline Prefix & Sub-code 1 & Postfix & $1 \ldots 1$ & 1 & $1 \ldots 1$ \\
\hline
\end{tabular}

Despreading Code

\begin{tabular}{|l|l|l|l|l|l|}
\hline $0 \ldots 0$ & Sub-code 1 & $0 \ldots 0$ & $0 \ldots 0$ & 1 & $0 \ldots \ldots 0$ \\
\hline
\end{tabular}

In order to reduce multipath interference the one-side ZCZ length $\mathrm{w}_{\min }$ must be larger than the maximum delay spread $\zeta_{\max }$ For conventional binary ZCZ codes, the ZCZ length must be $2^{\mathrm{k}}$, thus the one-side ZCZ length becomes $w_{\min }=2^{\left.\left[\log _{2}\left(\zeta_{\max }\right\rfloor+1\right)\right\rceil}$ .Thus set size of the conventional binary $\mathrm{ZCZ}$ codes is $\mathrm{S}_{\mathrm{con}}=\mathrm{C}_{\mathrm{L}} /$ $2^{\left\lceil\log _{2}\left(\zeta_{\max }+1\right)\right\rceil}$, where $C_{L}$ is the code length. However, for the $\mathrm{m}$-ZCZ code, $\mathrm{w}_{\min }$ can be any positive integer between 1 and $\mathrm{C}_{\mathrm{L}^{-}}$ 1. Hence, we set $w_{\min }=\left\lfloor\zeta_{\max }\right\rfloor^{+1}$ and the set size of $\mathrm{m}-\mathrm{ZCZ}$ code becomes $S_{m-z c z}=\left\lfloor\left(C_{L}-1\right) /\left(\left\lfloor\zeta_{\max }\right\rfloor+1\right)\right\rfloor$.

Table 1.Comparison of Correlation of $\mathbf{m}$-Sequences and $\mathbf{m}$ ZCZ Sequences

\begin{tabular}{|c|c|c|}
\hline $\begin{array}{l}\text { Sequence } \\
\text { Length }\end{array}$ & $\begin{array}{l}\text { Correlation of m- } \\
\text { Sequences }\end{array}$ & $\begin{array}{l}\text { Correlation of m- } \\
\text { ZCZ Sequences }\end{array}$ \\
\hline 3 & 0.333 & 0.01 \\
\hline 7 & 0.1428 & 0.009 \\
\hline 15 & 0.0667 & 0.0003 \\
\hline 31 & 0.0322 & 0.0001 \\
\hline 63 & 0.015873 & 0 \\
\hline
\end{tabular}

Table 1. shows the comparison of correlation of m-sequences and $\mathrm{m}-\mathrm{ZCZ}$ sequences. The correlation of $\mathrm{m}-$ Sequences and $\mathrm{m}-$ ZCZ Sequences are computed from equations (3) and (5).From this table it is observed that m-ZCZ Sequences have better cross correlation compared to that of m-Sequences. 


\section{SIMULATION MODEL}

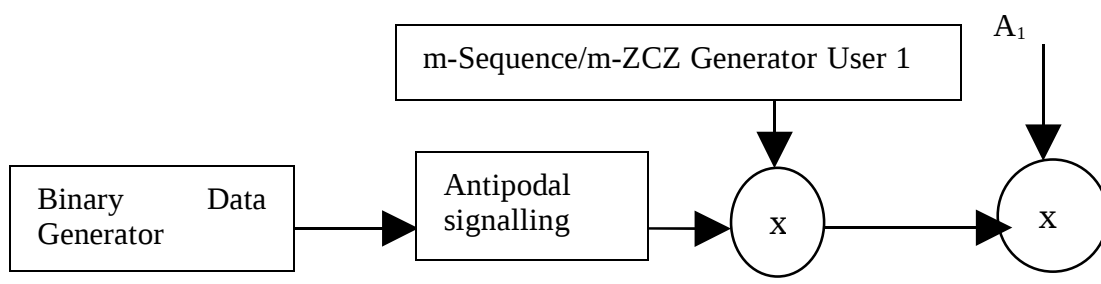

User 1

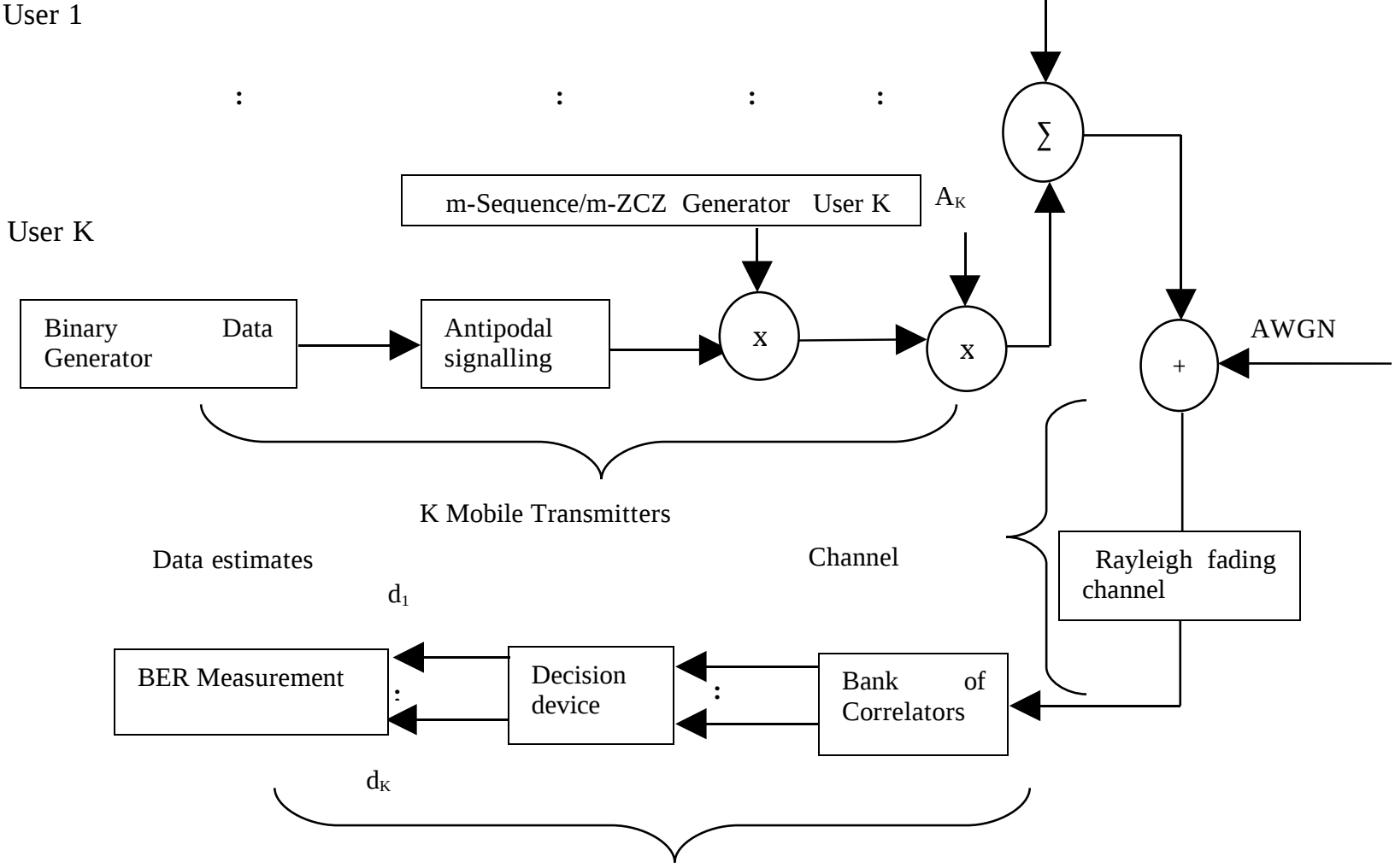

Base Station Receiver

\section{Figure 2. Simulation Model}

Figure 2. shows the simulation model of BER performance of $\mathrm{m}$ sequences and $\mathrm{m}-\mathrm{ZCZ}$ sequences in DS-CDMA System. The performance comparison of m-sequences and $\mathrm{m}-\mathrm{ZCZ}$ sequences are done using Monte Carlo simulations using Matlab with a confidence interval of $95 \%$. The binary data for the different users are generated using Bernoulli sequence generator. The random sequence binary of 0 's and 1 's are converted to antipodal form i.e., a sequence of $+1 \mathrm{~s}$ and -1 s. Each user's binary sequence is multiplied by the PN sequence of the corresponding user. The spreaded sequences are multiplied by the amplitude of each user. The amplitude values decide the power of each user. The transmitted sequences of different users are summed to represent the transmitted signal from different multiple mobile users. The resultant signal is then transmitted over an AWGN /Rayleigh flat fading channel [6].
AWGN Channel is considered because it is a stationary channel which affects the user's transmitted signal with background noise. Rayleigh fading channel is considered because it is a non stationary channel which affects the user's transmitted signal with multiuser interference. Both Channels have been considered in our simulation to account for various levels of multi user interference.

The received signal is jointly detected first by using a matched filter bank. The error between the detected sequence at the receiver and transmitted binary sequence is calculated. The number of errors divided by the total number of bits gives the BER. Throughout the simulation, it is assumed that

- the receiver has a perfect knowledge of the amplitude estimates of the different users

- $\quad$ spreading codes of the different users and the delays of the different transmitted signals arriving at the receiver are zero. 


\section{RESULTS AND DISCUSSION}

Monte Carlo simulations with a confidence interval of $95 \%$ have been run to simulate the Bit Error Rate (BER) performances of $\mathrm{m}$ - Sequences and m-ZCZ Sequences for different Bit Energy to Noise power spectral density ratio values $\left(E_{b} / N_{0}\right)$ and varying number of users in AWGN channel and Rayleigh fading conditions.

\section{Input Parameters}

\begin{tabular}{|l|l|}
\hline $\begin{array}{l}\text { Type of spreading } \\
\text { sequences }\end{array}$ & m-Sequences/m-ZCZ Sequences \\
\hline Frame length & 10000 \\
\hline Type of the channel & AWGN/ Rayleigh fading \\
\hline Ratio $E_{b} / N_{0}$ & $2-12 \mathrm{~dB}$ for AWGN channel \\
\hline Ratio $E_{b} / N_{0}$ & $\begin{array}{l}2-20 \mathrm{~dB} \text { for Rayleigh fading } \\
\text { channel }\end{array}$ \\
\hline
\end{tabular}

\section{Output Parameter}

The output parameter is the Bit Error Rate for varying number of Energy to Noise Density Ratio and for varying number of users. The BER is calculated using the following equation:

$$
B E R=\frac{\text { No of bits in error }}{\text { Total Number of bits transmitted }}
$$

\subsection{BER Performance m- Sequences/m-ZCZ Sequences in CDMA System}

The simulation results for BER performance and Capacity of DS-CDMA System using m-Sequences and m-ZCZ Sequences, are presented in Sections 4.1.1 to 4.1.8.

\subsubsection{Capacity of m-Sequence in AWGN channel}

Figure 3. shows the BER performance of DS-CDMA using mSequences in AWGN channel for $E_{b} / N_{0}=4 \mathrm{~dB}, 6 \mathrm{~dB}, 10 \mathrm{~dB}$ respectively with varying number of users. From this figure, it can be seen that the lowest BER is obtained when $E_{b} / N_{0}=10$ $\mathrm{dB}$. A BER of $10^{-3}$ is obtained for $10 \mathrm{~dB}$ curve at 140 users. At $E_{b} / N_{0}=4 \mathrm{~dB}$, BER is increased by 50 times at same number of users. Thus the BER value decreases as $E_{b} / N_{0}$ value increases. Also as number of users increases, BER increases.

\subsubsection{Capacity of m-ZCZ Sequences in AWGN channel}

Figure 4. shows the BER performance of DS-CDMA using mZCZ Sequences in AWGN channel for $E_{b} / N_{0}=4 \mathrm{~dB}, 6 \mathrm{~dB}, 10$ $\mathrm{dB}$ respectively with varying number of users. From this figure, it can be seen that the lowest BER is obtained when $E_{b} / N_{0}$ $=10 \mathrm{~dB}$. A BER of 0.0002 is obtained for $10 \mathrm{~dB}$ curve at 140 users. At $E_{b} / N_{0}=6 \mathrm{~dB}$, BER is increased by 75 times at same number of users.

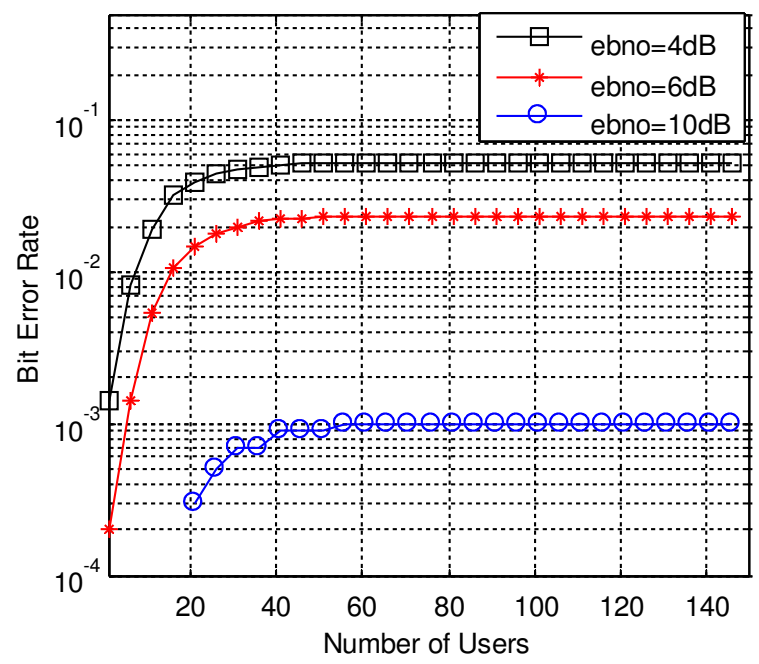

Figure 3.BER Performance of DS-CDMA using mSequences and $\mathrm{m}-\mathrm{ZCZ}$ Sequences in AWGN Channel

4.1.3 BER Performance of $m$-Sequences for varying Eb/No values in Rayleigh fading channel

Figure 5. shows the BER performance of $\mathrm{m}$-Sequences in Rayleigh fading channel for different for varying number of $E_{b} / N_{0}$. From this figure, it can be seen that the BER for 10 users, 100 users, 1000 users are 0.07, 0.1, 0.15 respectively when $E_{b} / N_{0}=4 \mathrm{~dB}$, BER for 10 users, 100 users, 1000 users are $0.0012,0.0025,0.005$ respectively when $E_{b} / N_{0}=9 \mathrm{~dB}$. Hence, as the number of users increases, the BER increases.

4.1.4. BER Performance of $m-Z C Z$ Sequences for varying Eb/No values in Rayleigh fading channel

Figure 6. shows the BER performance of m-ZCZ Sequences in Rayleigh fading channel for different for varying number of $E_{b} / N_{0}$. From this figure, it can be seen that the BER for 10

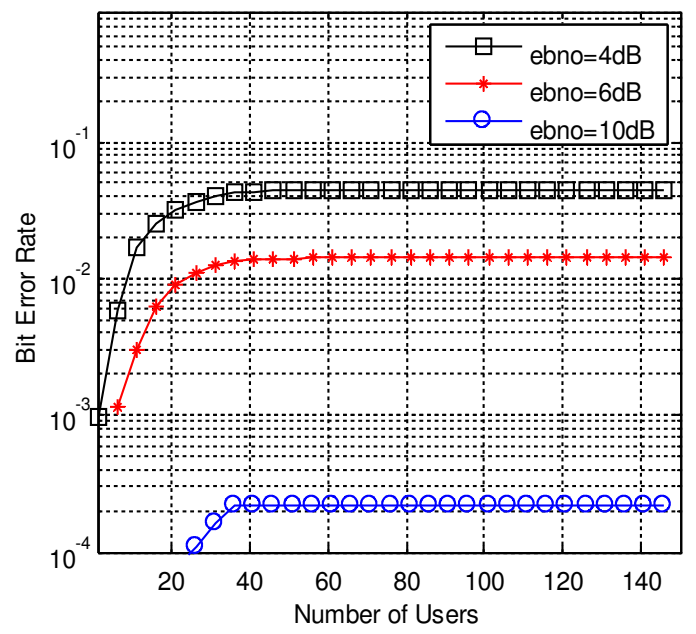

Figure 4.BER Performance of DS-CDMA using mSequences and $\mathrm{m}-\mathrm{ZCZ}$ Sequences in AWGN Channel 


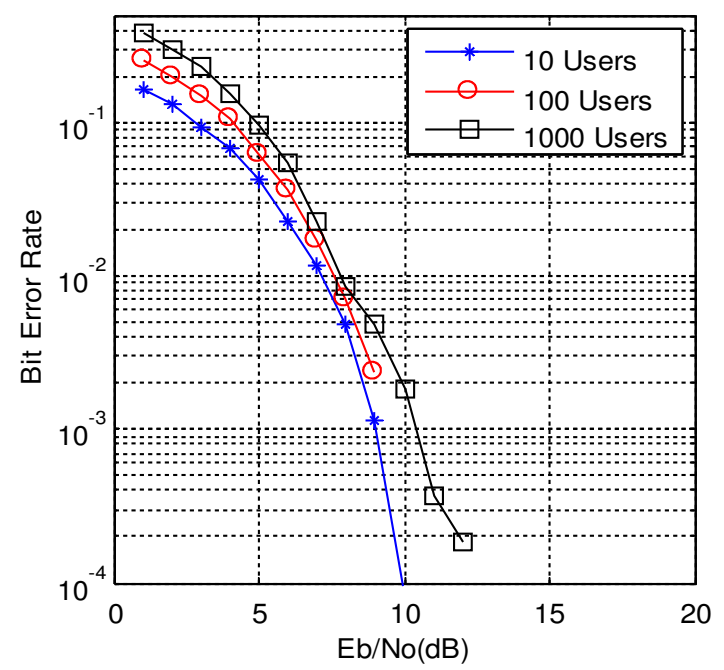

Figure 5.BER Performance of $\mathrm{m}$-Sequences in Rayleigh fading with 10, 100, 1000 users in DS-CDMA

users, 100 users, 1000 users are $0.0025,0.07,0.05$ respectively

when $E_{b} / N_{0}=4 \mathrm{~dB}$. The BER for 10 users, 100 users, 1000 users are $0.0002,0.005,0.0015$ respectively when $E_{b} / N_{0}=8$ $\mathrm{dB}$. Hence, as the number of users increases, the BER increases.

\subsubsection{Capacity of m-Sequences in Rayleigh channel}

Figure 7. shows the BER performance of CDMA using mSequences in Rayleigh channel for $E b / N o=2 d B, 10 \mathrm{~dB}, 20 \mathrm{~dB}$ respectively with varying number of users. From this figure, it can be seen that the lowest BER is obtained when $\mathrm{Eb} / \mathrm{No}=20$ $\mathrm{dB}$. A BER of $10^{-4}$ is obtained for $20 \mathrm{~dB}$ curve at 20 users and at $E_{b} / N_{0}=10 \mathrm{~dB}$, it is increased by 100 times at same number of users.

\subsubsection{Capacity of m-ZCZ Sequences in Rayleigh channel}

Figure 8. shows the BER performance of CDMA using m-ZCZ Sequences in Rayleigh channel for $\mathrm{Eb} / \mathrm{No}=2 \mathrm{~dB}, 10 \mathrm{~dB}, 20 \mathrm{~dB}$

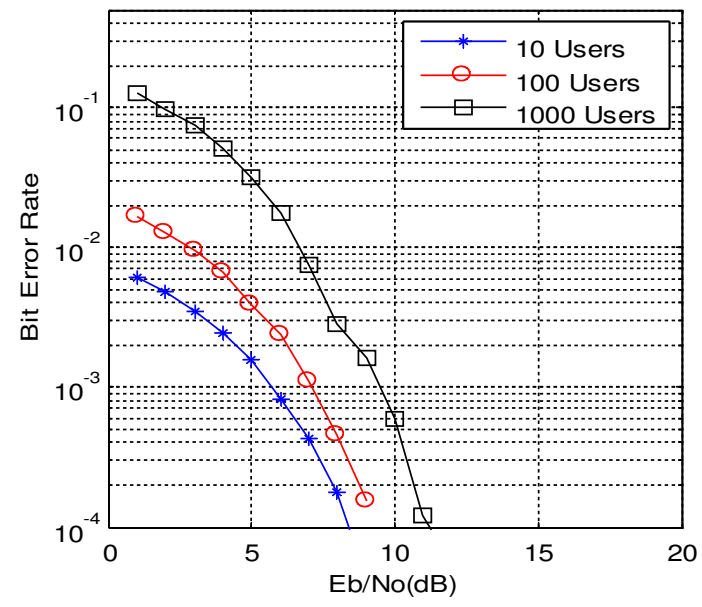

Figure 6.BER Performance of DS-CDMA using $\mathrm{m}-\mathrm{ZCZ}$ Sequences in Rayleigh fading with different users

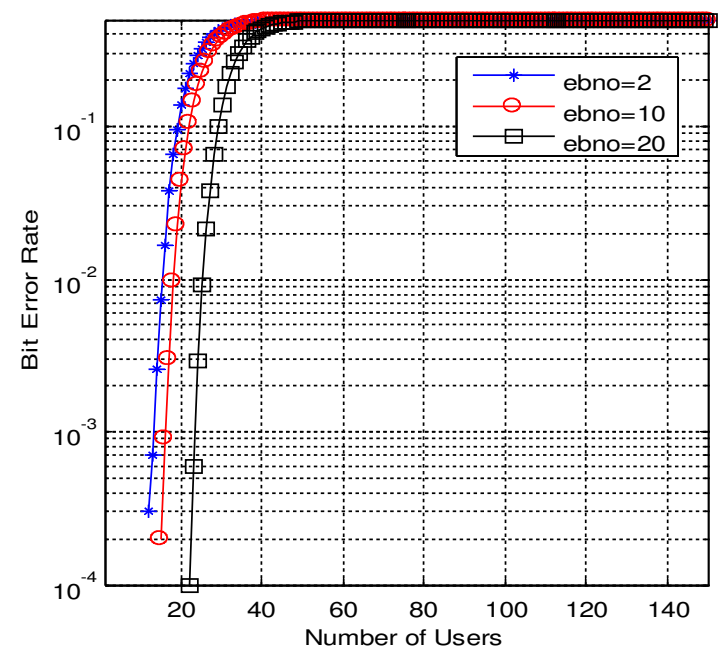

Figure 7.BER Performance of DS- CDMA using mSequences in Rayleigh Channel

respectively with varying number of users. From this figure, it can be seen that the lowest BER is obtained when $\mathrm{Eb} / \mathrm{No}=20$ $\mathrm{dB}$. A BER of 0.04 is obtained for $10 \mathrm{~dB}$ curve at 20 users but BER when $E_{b} / N_{0}=2 \mathrm{~dB}$ is increased by 5 times at same number of users.

4.1.7 Comparison of $m$-Sequences \& $m$-ZCZ Sequences in Rayleigh Channel in DS-CDMA System

Figure 9. shows a comparison between the m-sequences and proposed m-ZCZ Sequences in CDMA System for 1000 users under Rayleigh fading Conditions. At $E_{b} / N_{0}=18 \mathrm{~dB}$ it is found that m-ZCZ Sequences show a better BER performance by 10 times compared to that of m-Sequences because of their better Cross Correlation which validates the results shown in Table1.

Figure 10. shows a comparison between the m-sequences and proposed m-ZCZ Sequences in CDMA System under Rayleigh fading Conditions. At 50 users it is found that m-ZCZ Sequences show a better BER performance by 30 times

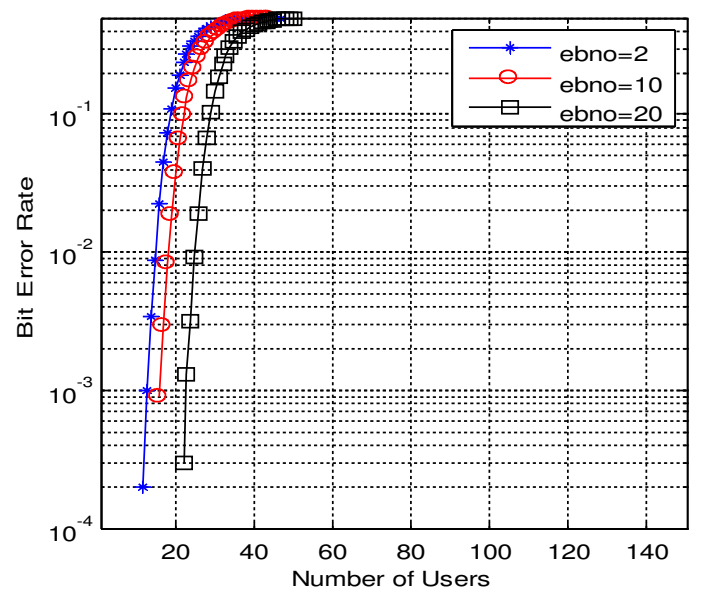

Figure8.BER Performance of DS- CDMA using m-ZCZ Sequences in Rayleigh Channel 


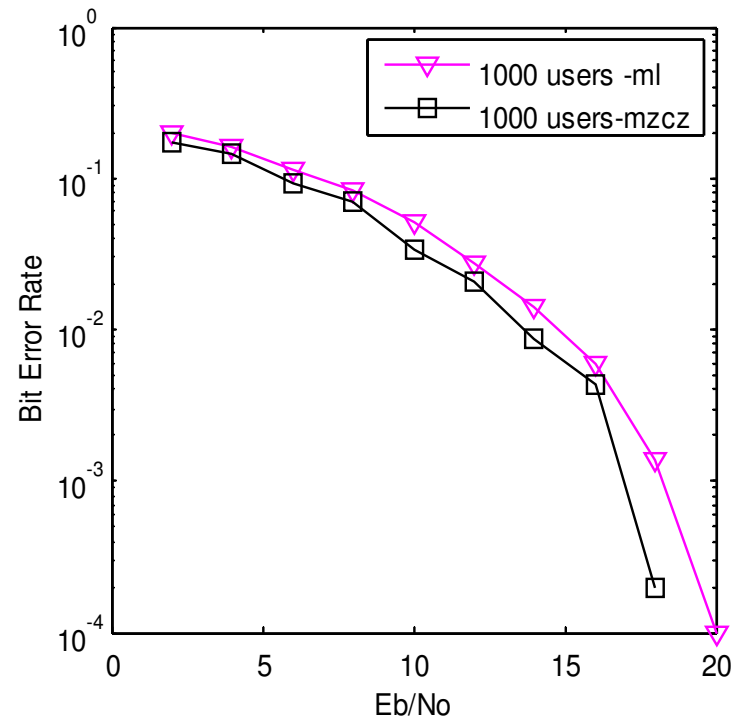

Figure 9. Comparison of BER performance of $\mathrm{m}$-Sequences and $\mathbf{m}$-ZCZ Sequences for 1000users in Rayleigh Channel

compared to that of m-Sequences because of their better Cross Correlation which validates the results shown in Table1.

\subsubsection{Comparison of m-Sequences \& $m$ - ZCZ Sequences in AWGN Channel in DS-CDMA System}

Figures 11. shows a comparison between the m-sequences and proposed m-ZCZ Sequences in CDMA System for 150 users under AWGN Channel Conditions. At 20 users when $\mathrm{Eb} / \mathrm{No}=4$ $\mathrm{dB}$ it is found that $\mathrm{m}-\mathrm{ZCZ}$ Sequences show a better BER performance by 20 times compared to that of m-Sequences because of their better Cross Correlation which validates the results shown in Table1.

\section{CONCLUSION}

In this paper Monte Carlo simulations have been run to compare BER performance of DS-CDMA System under AWGN and Rayleigh fading using $\mathrm{m}$-Sequences and $\mathrm{m}$-ZCZ Sequences.

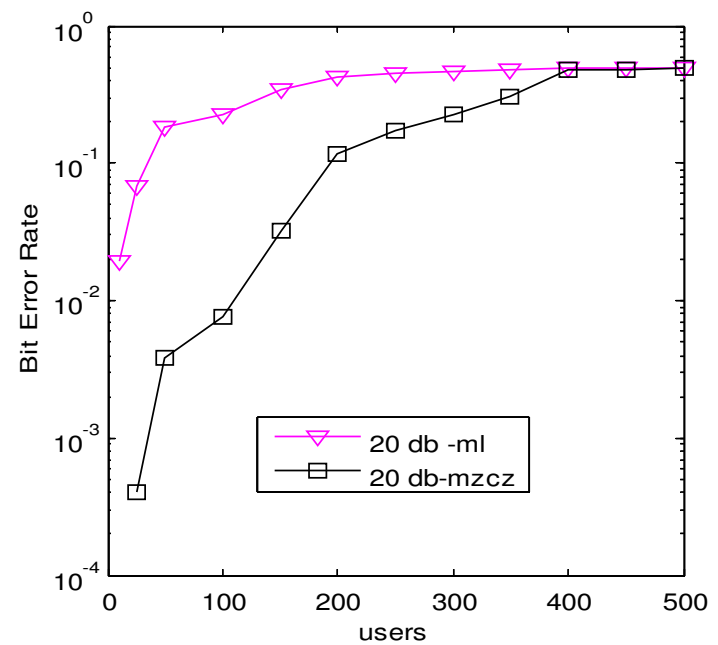

Figure10.Comparison of BER performance of $\mathbf{m}$ Sequences and $m-Z C Z$ Sequences for $E b / N_{0}=20 \mathrm{~dB}$ in Rayleigh

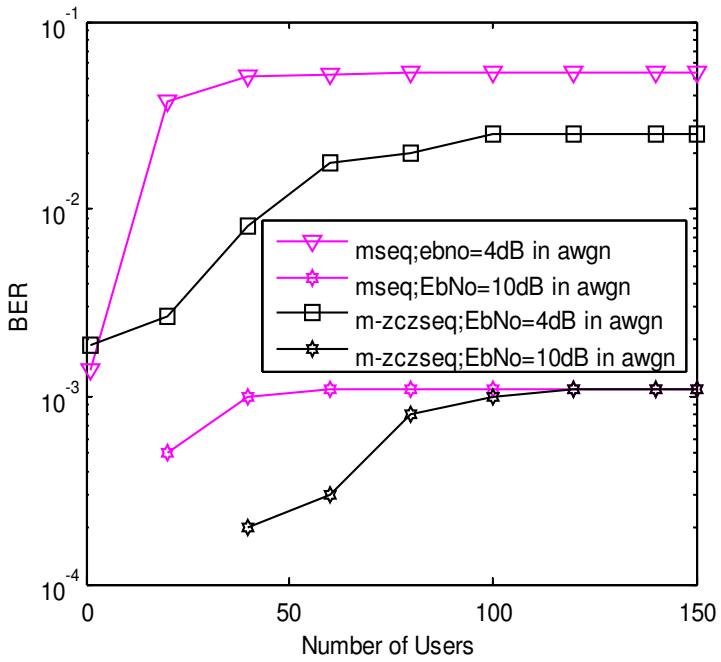

Figure11.Comparison of BER performance of mSequences and $\mathbf{m}$-ZCZ Sequences in AWGN Channel

It is observed that from the results, BER Performance of $\mathrm{m}-\mathrm{ZCZ}$ Sequences in CDMA is better than that of $\mathrm{m}$-Sequences because of the better Cross Correlation property of m-ZCZ Sequences.

\section{REFERENCES}

[1] Di Wu, Predrag Spasojevic and Ivan Seskar,”Orthogonal Variable Spreading factor codes with zero -correlation zone for TS UWB”IEEE Wireless Communications and Networking conference, 2(13-17): 807-812, March 2005.

[2] Ganlin Ye,Jing Li, Aiping Huang Hsiao-Hwa Chen, “A Novel ZCZ code based on m-sequences and its applications in CDMA systems", IEE Communication Letters,11(6), June 2007.

[3] Lifang Feng; Pingzhi Fan; Xiaohu Tang, “ A General Construction of OVSF Codes With Zero Correlation Zone”, IEEE Signal Processing Letters, 14(12): 908 - 911, December 2007.

[4] Rajeswari.A, "Investigations on Multiple Access Interference Cancellation Schemes in Code Division Multiple Access Based Cellular Systems”, Ph.D. Dissertation, Anna University, Chennai, 2006.

[5] Rathinakumar.A and Chaturvedi. A.K. "Mutually orthogonal sets of ZCZ sequences" ELECTRONICS LETTERS, 40 (18), September 2004.

[6] Raynold. L.P, Donald L.S and Milstein L.B, 'Theory of Spread Spectrum Communication - A Tutorial', IEEE Transactions on Communications. 30 (5): 855-884. May 1982.

[7] Theodore S.Rappaport, "Wireless Communication Principles and Practice Systems”, Prentice Hall,1997.

[8] H. Torii. H and M.N.N. Suehiro. M.N.N, "A new class of zero-correlation zone sequences," IEEE Trans. Inf. Theory, 50(3):559-565, March 2004.

[9] Xinmin Deng; Pingzhi Fan "Spreading sequence sets with zero correlation zone" Electronics Letters 36(11), 25: 993 -994, May 2000. 